\title{
Holographic fixing, readout, and storage dynamics in photorefractive materials
}

\author{
Amnon Yariv and Sergei Orlov \\ Department of Applied Physics, California Institute of Technology, Pasadena, California 91125
}

George Rakuljic* and Victor Leyva*

Accuwave Corporation, Santa Monica, California 90404

Received February 3, 1995

\begin{abstract}
Holographic gratings in photorefractive crystals that are based on charge redistribution inevitably decay as a result of ionic and electronic conduction. Under certain and restricted conditions these decay times can be acceptably long. Relevant decay rates and transient hologram field expressions are derived with special reference to $\mathrm{LiNbO}_{3}$. Some experimental data are presented.
\end{abstract}

Holographic data storage in photorefractive crystals is a topic of intense interest. It is driven by the prospects of the large storage capacity $\sim V / \lambda^{3}$ bits in a volume $V$. Two of the most important concerns in this field involve the lifetimes of fixed and developed holograms and their strengths. In general, the relevant physics is complicated, and one has to resort to numerical calculations. ${ }^{1}$ There exist practical situations, however, in which the time constants of the physical processes that are involved are sufficiently different, a situation that permits a closed-form solution for the grating strength transients during the fixing, developing, and storage. This is true in the case of doped $\mathrm{LiNbO}_{3}$ crystals used in our study.

A typical history of a hologram is shown in Fig. 1. In phase I an electronic grating, previously recorded, is heated to cause ion transport. This leads to a compensated grating that is represented by the net spacecharge field amplitude $E_{1}^{(1)}$. In phase II the grating is left in the dark or is exposed to infrared light, which is not photoactive. In many applications, however, the hologram is exposed to a reading light as in phase III. This light causes a partial redistribution of the trapped electrons culminating in a quasi-stable field $E_{1}^{(2)}$. This field will proceed to decay to zero under illumination as a result of ionic transport (phase IV).

The problem of grating dynamics has been considered by other investigators ${ }^{2-4}$ and, most notably, by Montemezzani et al. ${ }^{1}$ in a study of $\mathrm{KNbO}_{3}$. What differentiates our work is that, by taking advantage of the great disparity between the transport time constants that are involved in typical crystals, especially $\mathrm{LiNbO}_{3}$, we are able to obtain simple analytic expressions for the time-dependent field in each of the above-defined phases under realistic cases.

The dynamic variables are the mobile electron density $n_{e}$, the ion density $n_{i}$, and the trapped charge (ionized donor) density $N_{d}^{+}$. These are approximated in terms of (time and space) averages and small-signal time-varying amplitudes, i.e., $n_{e}=$ $n_{e 0}+\left[n_{e 1} \exp (-i K x)+\right.$ c.c. $]$, where $n_{e 1} \ll n_{e 0}, K$ is the spatial frequency of the grating, and $x$ is the spatial coordinate. A dependent variable is the space-charge field $E=E_{0}+\left[E_{1} \exp (-i K x)+\right.$ c.c. $]$ :

$$
E_{1}=\frac{i e}{\epsilon K}\left(N_{d 1}^{+}+n_{i 1}-n_{e 1}\right),
$$

which follows from the Gauss law. With the above definitions the linearized transport equations first formulated by Kukhtarev become

$$
\begin{aligned}
\frac{\partial N_{d 1}^{+}}{\partial t}= & -\left(\sigma / h \nu I_{0}+\beta\right) \frac{N_{d}}{N_{a}} N_{d 1}^{+}-\gamma_{e} N_{a} n_{e 1}, \\
\frac{\partial n_{e 1}}{\partial t}= & {\left[-\left(\sigma / h \nu I_{0}+\beta\right) \frac{N_{d}}{N_{a}}+\frac{\mu_{e} n_{e 0} e}{\epsilon}\right.} \\
+ & \left.i \frac{\kappa \sigma I_{0} K}{e}\right] N_{d 1}^{+}+\frac{\mu n_{e 0} e}{\epsilon} n_{i 1} \\
& -\left(\gamma_{e} N_{a}+\frac{\mu n_{e 0} e}{\epsilon}+D_{e} K^{2}+i \mu_{e} K E_{0}\right) n_{e 1}, \\
\frac{\partial n_{i 1}}{\partial t}= & -\left(\frac{\mu_{i} n_{i 0} e}{\epsilon}+D_{i} K^{2}+i \mu_{i} K E_{0}\right) n_{i 1} \\
& +\frac{\mu_{i} n_{i 0} e}{\epsilon}\left(n_{e 1}-N_{d 1}^{+}\right),
\end{aligned}
$$

where the total current is taken as a sum of ionic and electronic drift, diffusion currents, and a photovoltaic current density $\kappa \sigma\left(N_{d}-N_{d}^{+}\right) I_{0}$.

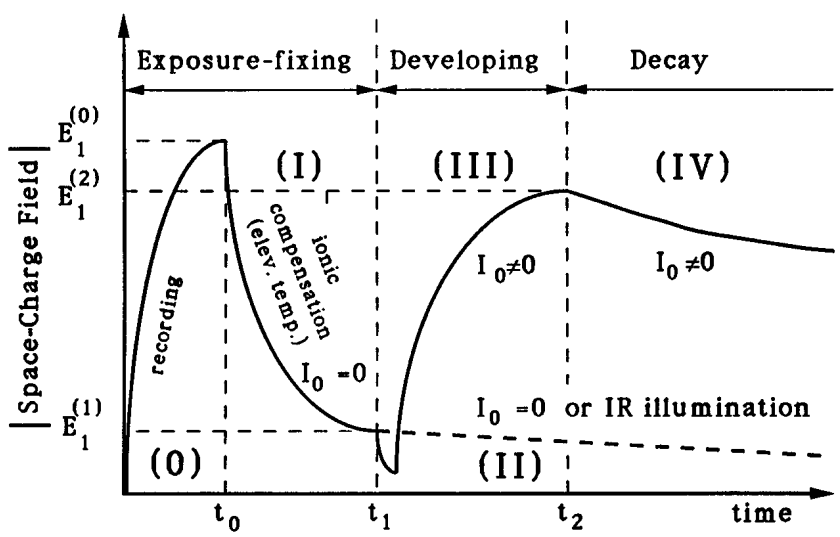

Fig. 1. Typical life history of a hologram in a photorefractive material. 
In Eqs. (2)-(4) $I_{0}$ is the average optical intensity, $\sigma N_{d}^{0}$ is the absorption coefficient, and $\beta$ is the thermal excitation rate. $\mu_{e}$ and $\mu_{i}$ are the mobilities of the electrons and ions, and we assumed that $D / \mu=k T / e$, where $k$ is the Boltzmann in constant. $\kappa$ is the photovoltaic coefficient. The density of neutral donors is $N_{d}^{0}$, the total donor density is $N_{d}=N_{d}^{0}+N_{d}^{+}$, and $N_{d 0}^{+}=N_{a}$ is the density of deep traps.

A major feature of our analysis is the inequality $n_{e 1} \ll N_{d 1}^{+}, n_{i 1}$ so that $n_{e 1}$ can be neglected in Eqs. (1) and (4) but not otherwise. This inequality results from the fact that the electron-trapping rate $\gamma_{e} N_{a} \sim$ $10^{7}-10^{9} \mathrm{~s}^{-1}$ exceeds by many orders of magnitude any other rate. This causes the electrons to reach an essentially instantaneous (i.e., within $t \leq 10^{-7} \mathrm{~s}$ ) local equilibrium with $N_{d 1}^{+}$and $n_{i 1}$. We obtain the equilibrium value of $n_{e 1}$ by setting the left-hand side of Eq. (3) equal to zero. This enables us to eliminate $n_{e 1}$ from the mathematics (but not from the physics). In what follows we consider the solutions of Eqs. (1)-(4) in each of the phases of Fig. 1.

Phase I: This phase involves compensation of the electronic space-charge field by ionic transport. The space-charge field is given by

$$
\begin{aligned}
E_{1}^{(\mathrm{I})}(t)= & E_{1}^{(0)}(0)\left\{\frac{D_{i} K^{2}+i K \mu_{i} E_{0}}{\omega_{i}+D_{i} K^{2}+i K \mu_{i} E_{0}}\right. \\
& +\frac{\omega_{i}}{\omega_{i}+D_{i} K^{2}+i K \mu_{i} E_{0}} \\
& \left.\times \exp \left[-\left(\omega_{i}+D_{i} K^{2}+i K \mu_{i} E_{0}\right) t\right]\right\},
\end{aligned}
$$

where $\omega_{i}$ is the ionic dielectric relaxation frequency $\omega_{i}=\left(e \mu_{i} n_{i 0} / \epsilon\right), D_{i}$ is the ionic diffusion coefficient, and $E_{1}^{(0)}(0)$ is the space-charge field of the initially recorded (phase 0 in Fig. 1) electronic grating. The steady-state stabilized field $E_{1}^{(1)}$ is

$$
E_{1}^{(1)}=\frac{D_{i} K^{2}+i K \mu_{i} E_{0}}{\omega_{i}+D_{i} K^{2}+i K \mu_{i} E_{0}} E_{1}^{(0)}
$$

In data storage applications the aim of this stage is to culminate in as perfect a compensation as possible, i.e., $n_{i 1}\left(t_{1}\right) \approx-N_{d 1}^{+}\left(t_{0}\right)$ or equivalently $E_{1}^{(1)} \ll E_{1}^{(0)}$. This requires, according to Eq. (6), that $\omega_{i} \gg D_{i} K^{2}$ or equivalently $n_{i 0} \gg\left(\epsilon k T / e^{2}\right) K^{2}$. The latter condition sets the lower boundary for the optimized density of the free ions in a crystal used for holographic data storage. Further increase of $n_{i 0}$ does not improve the fixing efficiency but rather decreases the possible storage time because the ionic relaxation frequency $\omega_{i}$ is proportional to $n_{i 0}$ at any temperature. The above results are applicable, in their basic features, also in the case when the recording phase (0) and the ionic compensation phase (I) are simultaneous. If the ionic compensation takes place in the presence of light under open circuit conditions, then $E_{0}=-E_{\text {p.v. }}=-\kappa \gamma_{e} N_{a} / e \mu_{e}$.

Phase II: This phase consists of a slow decay in the dark of the ionically compensated electronic grating. This decay is due to thermally excited electrons that proceed to drift and diffuse in order to screen the residual field $E_{1}^{(1)}$. This process is accompanied by the much faster (at elevated temperature ${ }^{5,6}$ ) ad- justment by transport of ions so that any moment $n_{i 1} / N_{d 1}^{+}=-\omega_{i} /\left(\omega_{i}+D_{i} K^{2}\right)$. The space-charge field (i.e., the grating amplitude) decays as

$$
\begin{aligned}
E_{1}^{(\mathrm{II})}(t)= & \frac{i e}{\epsilon K}\left[N_{d 1}^{+}(t)+n_{i 1}(t)\right]=E_{1}^{(0)} \frac{D_{i} K^{2}}{\omega_{i}+D_{i} K^{2}} \\
& \times \exp \left[-\omega_{e}\left(\frac{D_{i} K^{2}}{D_{i} K^{2}+\omega_{i}}+K^{2} d^{2}\right) t\right] \\
\omega_{e}= & \frac{e \mu_{e} n_{e 0}}{\epsilon}=\frac{e \mu_{e}\left(\sigma / h \nu I_{0}+\beta\right)\left(N_{d}-N_{a}\right)}{\epsilon \gamma_{e} N_{a}}, \\
d^{2} \equiv & \frac{N_{d} k T \epsilon}{N_{a}\left(N_{d}-N_{a}\right) e^{2}},
\end{aligned}
$$

where $d$ is the electronic Debye screening length. The decay rate $\omega_{\text {decay }}^{(\text {II) }}=\omega_{e}\left[D_{i} K^{2} /\left(\omega_{i}+D_{i} K^{2}\right)+K^{2} d^{2}\right]$ is equal to the electronic dielectric relaxation frequency $\omega_{e}\left(\right.$ at $\left.I_{0}=0\right)$ multiplied by the ionic screening factor plus a term that is due to diffusion of electrons. The above results are for $E_{0}=0$. Otherwise, we replace $D_{i} K^{2}$ everywhere with $D_{i} K^{2}+i K \mu_{i} E_{0}$ and $K^{2} d^{2}$ with $K^{2} d^{2}+i K \mu_{e} E_{0}$.

Experimental observations ${ }^{5}$ reproduced in Fig. 2 show the dark decay of holographic gratings recorded in $\mathrm{Fe}: \mathrm{LiNbO}_{3}$ at elevated temperature. The two curves of Fig. 2 were obtained with two different initial diffraction efficiencies in otherwise similar crystals. Two distinct stages of the process (fast and slow) can be identified as fast ionic compensation (phase I) and the much slower thermal decay of the electronic grating partially screened by the mobile ions (phase II). From the data of Fig. 2 we obtain $\left|E\left(t_{1}\right) / E\left(t_{0}\right)\right| \approx 0.1$, which according to Eq. (5) corresponds to $\omega_{i} \approx 9 D_{i} K^{2}$ when $\Lambda=2 \pi / K=0.34 \mu \mathrm{m}$.

Phase III: This stage consists of the developing (revealing) of the compensated grating by continuous illumination $\left(I_{0} \neq 0\right)$ at or near room temperature. This phase also describes what happens when we read the hologram. This causes an increase in the electronic conductivity so that it greatly exceeds that of

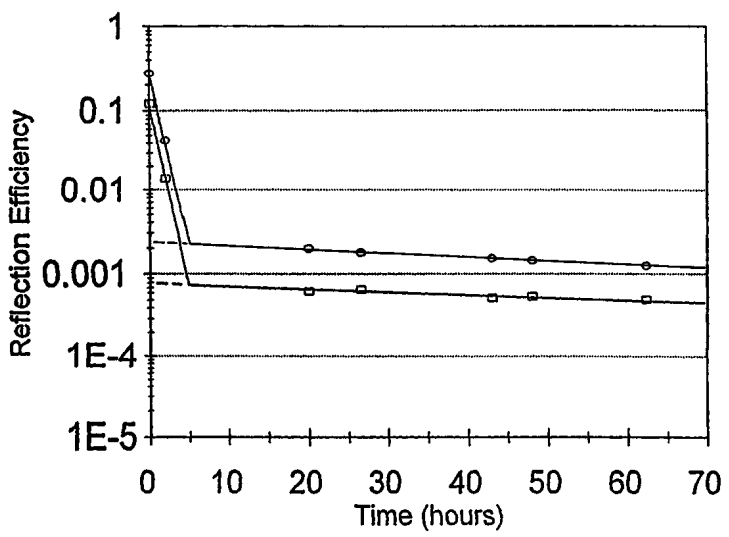

Fig. 2. Diffraction efficiency versus time of two holograms recorded and stored in Fe-doped $\mathrm{LiNbO}_{3}$ at $110{ }^{\circ} \mathrm{C}$. The grating spacing is $\sim 0.34 \mu \mathrm{m}$. The initial $(t=0)$ diffraction efficiencies were $\sim 30 \%$ (upper curve, circles) and $\sim 12 \%$ (lower curve, squares). Because of ionic compensation (fast stage of the decay) the reflection efficiency decreases by a factor of $\sim 100$ for both holograms. It continues to decay further in the dark at a much slower rate because of conduction by thermally excited electrons. 
the ions $\left(\sigma_{e} \gg \sigma_{i}\right)$. In this stage it is crucial to include the photovoltaic effect when the crystal possesses it. We use the commonly used short-circuit condition $\left(E_{0}=0\right)$ and allow for the full de photovoltaic current $\kappa \alpha I_{0}\left(N_{d}-N_{a}\right)$ to flow.

The resulting space-charge holographic field during phase III is

$$
\begin{aligned}
E_{1}^{(\mathrm{IIII})}(t)= & \frac{i e}{\epsilon K}\left\{\frac{E_{d}-i E_{\mathrm{p} . \mathrm{v} .} N_{a} / N_{d}}{E_{d}+E_{q}-i E_{\text {p.v. }} N_{a} / N_{d}} n_{i 1}\left(t_{1}\right)\right. \\
& +\left[N_{d 1}^{+}\left(t_{1}\right)+\frac{E_{q}}{E_{d}+E_{q}-i E_{\text {p.v. }} N_{a} / N_{d}} n_{i 1}\left(t_{1}\right)\right] \\
& \left.\times \exp \left(-\omega_{i} t\right)\right\} \\
\omega_{1} \equiv & \omega_{e}\left[1+K^{2} d^{2}-i \frac{E_{\text {p.v. }}}{E_{q}}\left(\frac{N_{a}}{N_{d}}\right)\right]
\end{aligned}
$$

where $E_{\text {p.v. }} \equiv \kappa \sigma / h \nu I_{0}\left(N_{d}-N_{a}\right) /\left(e \mu_{e} n_{e 0}\right)=\kappa \gamma_{e} N_{a} / e \mu_{e}$ is the characteristic photovoltaic field, $E_{q}=e N_{a}(1-$ $\left.N_{a} / N_{d}\right) / \epsilon \mathrm{K}$ is the limiting space-charge field, and $E_{d} \equiv$ $D_{e} K / \mu_{e}=K k T / e$ is the diffusion field.

Equation (8) predicts a quasi-stabilized field at the end of phase III in the presence of illumination whose value is

$$
E_{1}^{(2)}=\frac{i e}{\epsilon K} n_{i 1}\left(t_{1}\right) \frac{E_{d}-i E_{\text {p.v. }} N_{a} / N_{d}}{E_{d}+E_{q}-i E_{\text {p.v. }} N_{a} / N_{d}},
$$

independent of the initial trapped charge grating $N_{d 1}^{+}\left(t_{1}\right)$. This field can approach the original field $E_{1}^{(0)}$, provided that $n_{i 1}\left(t_{1}\right) \approx-N_{d 1}^{+}\left(t_{0}\right)$ and $E_{1}^{(2)} \approx$ $(i e / \epsilon K) n_{i 1}\left(t_{1}\right)$. The first condition is satisfied when the ionic compensation in phase I is nearly complete, which according to Eq. (6) happens when $\omega_{i} \gg D_{i} K^{2}$, i.e., the density of ions is sufficiently large $n_{i 0} \gg\left(\epsilon k T / e^{2}\right) K^{2}$. The second condition requires, according to Eq. (9), that either $E_{d} \gg E_{q}$ (high spatial frequencies, i.e., $K \gg K_{e} \equiv 1 / d$ ) or $E_{\text {p.v. }} \gg E_{q}$ (strong photovoltaic effect).

Phase IV: This phase relates directly to the lifetime of the hologram in actual applications. It involves the decay of the ionic backbone grating as a result of ion transport. In phase III we neglected ionic transport. [This was justified because the duration of that phase is short compared with the ionic decay time $\left(\omega_{i}+D_{i} K^{2}\right)^{-1}$.] This assumption resulted in a stabilized space-charge field (hologram), as given by Eq. (9). This field proceeds to decay to zero because of the ionic transport that erases the ionic backbone charge $n_{i 1}$. The key physical assumption here is that this process is sufficiently slow that while it takes place the faster electronic transport in the presence of light causes the trapped charge density $N_{d 1}^{+}(t)$ to be always in equilibrium with $n_{i 1}$. This equilibrium ratio is obtained from Eqs. (4) and (8) for $t \rightarrow \infty N_{d 1}^{+}(t) / n_{i 1}(t)=$ $-E_{q} /\left(E_{d}+E_{q}-i E_{\text {p.v. }} N_{a} / N_{d}\right)$. With this last condition we obtain from Eq. (4)

$$
n_{i 1}^{(\mathrm{IV})}(t)=n_{i 1}\left(t_{2}\right) \exp \left\{-\left[\omega_{i}\left(\frac{\omega_{1}-\omega_{e}}{\omega_{1}}\right)+D_{i} K^{2}\right] t\right\}
$$

for the short-circuit case. We recall that $n_{i 1}\left(t_{2}\right) \approx$ $n_{i 1}\left(t_{1}\right)$ because phase III is too short for significant ionic transport.
We obtain the corresponding grating fields from Eq. (9) by neglecting the transient term and replacing $n_{i}\left(t_{1}\right)$ by its solution [Eq. (10)]. The result is that during phase IV the hologram decays to zero because of ionic transport, which is slowed because of partial electronic compensation:

$$
\begin{aligned}
E_{1}^{(\mathrm{IV})}(t)= & \frac{i e}{\epsilon K}\left(\frac{E_{d}-i E_{\text {p.v. }} N_{a} / N_{d}}{E_{d}+E_{q}-i E_{\text {p.v. }} N_{a} / N_{d}}\right) n_{i 1}\left(t_{1}\right) \\
& \times \exp \left\{-\left[\omega_{i}\left(\frac{\omega_{1}-\omega_{e}}{\omega_{1}}\right)+D_{i} K^{2}\right] t\right\} .
\end{aligned}
$$

A few main conclusions are worth emphasizing:

(1) In infrared applications, such as in wavelengthmultiplexing filters ${ }^{7}$ or under dark storage conditions, the (phase II) decay as described by Eq. (7) is due to thermal electronic transport slowed by ionic screening. This process at room temperatures can possess projected lifetimes in our $\mathrm{LiNbO}_{3}$ crystals of $\sim 100$ years. ${ }^{5,6}$

(2) A near-perfect development [i.e., $E_{1}\left(t_{2}\right) \approx E_{1}\left(t_{0}\right)$ ] can be obtained. See the conditions described after Eq. (9).

(3) Under illumination (phase IV) the hologram is quasi-stabilized [Eq. (9)]. This stabilized value can approach the initial value $E_{1}\left(t_{2}\right)$, provided that the screening of the residual ionic grating by electronic grating is small, which according to Eq. (9) happens when $E_{d} \gg E_{q}$ or $E_{\text {p.v. }} \gg E_{q}$. The residual decay is due to the transport of the ionic charge backbond. Major reduction of the ionic conductivity at the operating temperature is necessary to bring this decay rate to a commercially acceptable range of, say, lifetimes of $\geq 10$ years.

A. Yariv and S. Orlov acknowledge the support of an Advanced Research Projects Agency University Research Initiative grant (L. N. Durvasula), the U.S. Army Research Office (R. Guenther), and the National Institute of Standards and Technology. V. Leyva and G. Rakuljic acknowledge the support of the National Institute of Standards and Technology through the ATP program.

A. Yariv is a consultant with Accuwave Corporation.

*Present address, Arroyo Optics, Inc., Santa Monica, California 90404.

\section{References}

1. G. Montemezzani, M. Zgonik, and P. Günter, J. Opt. Soc. Am. B 10, 171 (1993).

2. S. Orlov, D. Psaltis, and R. R. Neurgaonkar, Appl. Phys. Lett. 63, 2466 (1993).

3. M. Carrascosa and F. Agullo-Lopez, J. Opt. Soc. Am. B 7, 2317 (1990).

4. R. Matull and R. A. Rupp, J. Phys. D 21, 1556 (1988).

5. D. L. Staebler, W. J. Burke, W. Phillips, and J. J. Amodei, Appl. Phys. Lett. 26, 182 (1975).

6. A. Yariv, V. Leyva, and G. A. Rakuljic, in Technical Digest, 1994 IEEE Nonlinear Optics, Materials, Fundamentals, and Applications (Institute of Electrical and Electronics Engineers, New York, 1994), paper PD6.

7. V. Leyva, G. A. Rakuljic, and B. O'Conner, Appl. Phys. Lett. 65, 1079 (1994). 